\title{
Segurança do paciente e a pandemia causada pelo SARS-CoV-2
}

\author{
Bárbara do Nascimento Caldas ${ }^{1}$ \\ Eliana Auxiliadora Magalhães Costa ${ }^{2}$ \\ Lenice Gnocchi da Costa Reis ${ }^{3}$ \\ Mariluce Karla Bomfim de Souza ${ }^{4}$ \\ Ana Cristina Souto 5
}

1 Médica sanitarista do Instituto Nacional de Cardiologia (INC), doutora em Saúde Pública pela Escola Nacional de Saúde Pública Sergio Arouca (ENSP) da Fundação Oswaldo Cruz (Fiocruz), mestre em Administração de Empresas pela Escola de Administração de Empresas de São Paulo (EAESP) da Fundação Getúlio Vargas (FGV), graduada em Medicina pela Universidade Federal Fluminense (UFF).

2 Professora titular do Departamento de Ciências da Vida da Universidade do Estado da Bahia (UNEB), doutora em Saúde Pública pelo Instituto de Saúde Coletiva (ISC) da Universidade Federal da Bahia (UFBA), mestre em Organização de Serviços de Saúde pela UFBA, graduada em Enfermagem pela Universidade Católica do Salvador (UCSal).

3 Pesquisadora da Escola Nacional de Saúde Pública Sérgio Arouca (ENSP) da Fundação Oswaldo Cruz (Fiocruz), doutora em Saúde Pública pela ENSP da Fiocruz, mestre em Ciências da Saúde pela Fiocruz, graduada em Medicina pela Universidade Estadual do Rio de Janeiro (UERJ).

4 Professora adjunta do Instituto de Saúde Coletiva (ISC) da Universidade Federal da Bahia (UFBA), pós doutorado pela Universidad Complutense de Madrid (UCM/ES), doutora em Saúde Pública pelo ISC da UFBA, mestre em Saúde Coletiva pela Universidade Estadual de Feira de Santana (UEFS), mestre em Enfermagem pela UFBA, graduada em Enfermagem pela Universidade Estadual de Santa Cruz (UESC).

5 Professora associada do Instituto de Saúde Coletiva (ISC) da Universidade Federal da Bahia (UFBA), doutora em Saúde Pública pelo ISC da UFBA, mestre em Saúde Comunitária pelo ISC da UFBA, graduada em Nutrição pela Universidade Federal da Paraíba (UFPB).

CALDAS, B. do N.; COSTA, E. A. M.; REIS, L. G. da C.; SOUZA, M. K. B. de; SOUTO, A. C. Segurança do paciente e a pandemia causada pelo SARS-CoV-2. In: BARRETO, M. L.; PINTO JUNIOR, E. P.; ARAGÃO, E.; BARRAL-NETTO, M. (org.). Construção de conhecimento no curso da pandemia de COVID-19: aspectos biomédicos, clínico-assistenciais, epidemiológicos e sociais. Salvador: Edufba, 2020. v. 2. 


\section{Introdução}

Problemas de segurança do paciente configuram-se como questão mundial de saúde pública. Dados apontam que erros no cuidado de saúde representam a terceira causa de morte nos Estados Unidos, responsável por $400 \mathrm{mil}$ óbitos/ano, atrás somente de doenças cardiovasculares e câncer. (MAKARY; DANIEL, 2016) No Reino Unido, estima-se que, em média, um incidente com dano ao paciente é notificado a cada 35 segundos. (WHO, 2017) No Brasil, calcula-se que os Eventos Adversos (EA) consomem de R\$ 5,19 a 15,5 bilhões/ano, montante relativo apenas à saúde suplementar. (COUTO; PEDROSA; ROSA, 2016)

A ocorrência de EA, além das consequências econômicas, traz importantes repercussões para pacientes, familiares, serviços de saúde e para toda a sociedade. Por vezes, também para os profissionais de saúde envolvidos, considerados por alguns autores como as "segundas vítimas”, por apresentarem reações emocionais, cognitivas e comportamentais, o que requer, portanto, a adoção de estratégias de enfrentamento para esse agravo sanitário. (SCOTT et al., 2009; WU, 2000)

Nos últimos anos, formuladores de políticas, profissionais de saúde e pesquisadores vêm investindo no desenho, implementação e estudos de iniciativas para melhoria 
da segurança do paciente. No entanto, todo esse investimento ainda não representou resultados substanciais de modo a reduzir riscos aos quais pacientes estão expostos nos serviços de saúde. Esses riscos foram potencializados com a eclosão da pandemia da COVID-19 que demandou das organizações de saúde e de seus profissionais agilidade para mudanças nos processos de trabalho, seja pela necessidade de adoção de novos arranjos de cuidado exigidos ou pela intensificação do uso de procedimentos diagnósticos e terapêuticos. A repercussão mais imediata foi a introdução de possibilidades para desorganização das práticas assistenciais e de novos perigos à segurança do paciente.

Este capítulo inicia-se com uma síntese acerca dos principais conceitos, práticas e aspectos político-institucionais relacionados à segurança do paciente no Brasil. Em seguida, sintetiza dados publicados sobre essa temática durante a pandemia da COVID-19, particularizando estratégias organizacionais para gestão do risco e finaliza com reflexões sobre os efeitos da pandemia para a segurança do paciente no Brasil.

\section{Segurança do paciente: principais conceitos e noções}

A maior parte da discussão relacionada à segurança do paciente envolve a noção de dano - doença, dano ou lesão, sofrimento, incapacidade ou disfunção e morte - que pode ser físico, social ou psicológico. O dano associado ao cuidado de saúde é aquele surgido por ou associado a planos ou ações realizadas durante o cuidado de saúde e não a um problema de saúde ou uma doença de base.

Há muitas décadas sabe-se que o cuidado de saúde também pode gerar doença (iatrogenia). Mas foi a partir do relatório To err is human publicado pelo Instituto de Medicina (IOM) dos Estados Unidos, que o tema da segurança do paciente ganhou visibilidade. 
Ao reconhecer a magnitude do problema de segurança do paciente a nível global, a Organização Mundial da Saúde (OMS) estabeleceu, em 2004, a Aliança Mundial para a Segurança do Paciente, com o propósito de identificar e estabelecer prioridades nessa área e contribuir para uma agenda mundial de enfrentamento desse problema.

No começo dos anos 2000, diversos termos foram usados para se referir a problemas de segurança no cuidado de saúde. (MENDES, 2019) Para proporcionar uma linguagem comum, uma das primeiras iniciativas da Aliança foi a elaboração de uma Classificação Internacional de Segurança do Paciente (Cisp). (RUNCIMAN et al., 2009) De acordo com essa classificação, segurança do paciente é definida como redução, a um mínimo aceitável, do risco de dano desnecessário associado ao cuidado de saúde. (RUNCIMAN et al., 2009) Mínimo aceitável se refere às noções coletivas de conhecimento atual, recursos disponíveis e o contexto no qual o cuidado é prestado e considerado frente ao risco, de não tratar ou de um tratamento alternativo. O termo "desnecessário" na definição de segurança serve para distinguir entre eventos adversos e complicações. Nenhuma intervenção em saúde é livre de risco. Muitas, inclusive, implicam efeitos danosos, como desconforto e sofrimento, mas esses riscos são avaliados em comparação aos benefícios esperados, por exemplo, o tratamento quimioterápico. Nesses casos, tais efeitos não costumam ser considerados dano ao paciente.

Um erro é uma falha em executar um plano de ação como pretendido - por exemplo, falha na administração de um medicamento - ou a aplicação de um plano incorreto - por exemplo, erro diagnóstico. (RUNCIMAN et al., 2009) Erros são, por definição, não intencionais, enquanto violações são atos intencionais que, no cuidado de saúde, não costumam ser maliciosas, mas podem se tornar rotineiras e automáticas em certos contextos. Um exemplo de violação rotineira é a não adesão à higienização das mãos por 
profissionais de saúde. Erros não levam necessariamente ao dano, mas podem contribuir para sua ocorrência.

Incidente relacionado ao cuidado de saúde, ou apenas incidente, é um evento ou circunstância que poderia ter resultado, ou resultou, em dano desnecessário ao paciente. Os incidentes classificam-se como:

- Circunstância notificável - houve potencial significativo para o dano, mas o incidente não ocorreu (por exemplo, identificação de um desfibrilador que não está funcionando na vistoria da sala de emergência);

- Near miss - incidente que não atingiu o paciente (por exemplo, um erro de prescrição que é corrigido antes da dispensação do medicamento);

- Incidente sem dano - incidente que atingiu o paciente, mas não causou dano (por exemplo, administração de medicamento ao paciente errado que não causou dano);

- Incidente com dano (EA) - incidente que resulta em dano ao paciente (por exemplo, queda do leito com fratura dos ossos da face).

Outro conceito importante é evitabilidade. Nem todo EA pode ser evitado dado o conhecimento, informação e estado da arte do cuidado de saúde no momento do incidente. Por exemplo, uma reação alérgica a um medicamento administrado pela primeira vez é um EA, mas seria considerado um EA não evitável, pois não havia informação anterior sobre a alergia do paciente. Porém, qualquer administração subsequente desse medicamento a esse paciente seria considerada um erro de medicação evitável, constituindo clara falha de segurança.

Muitas vezes após a ocorrência de um erro ou EA, as pessoas tendem a atribuir como principal causa - se não a única - a ação do profissional de saúde diretamente envolvido no incidente. No entanto, a segurança do paciente analisa a ocorrência de um 
incidente orientada pela abordagem sistêmica que entende que tanto os sucessos, quanto as falhas no cuidado de saúde, são produtos multifatoriais. (REIS, 2019)

A distinção entre falhas ativas e falhas latentes (REASON, 2000) é especialmente útil na compreensão da ocorrência de incidentes. Falhas ativas - erros ou violações - são cometidas por pessoas envolvidas diretamente com a operação dos processos no cuidado de saúde, e, geralmente, são consequências de condições antecedentes existentes nas organizações - condições ou falhas latentes. As condições latentes resultam de decisões de pessoas envolvidas com o desenho e controle dos processos organizacionais ao longo do tempo - gerentes, diretores. (REASON, 2000)

Reason (2000) destaca que defesas e barreiras são componentes-chave em um sistema seguro. Elas podem ser produtos de engenharia, como os alarmes e as barreiras físicas, ou dependerem da atuação de pessoas, como cirurgiões e anestesistas; têm como função defender potenciais vítimas dos perigos locais. Porém, essas barreiras não são intactas e se, momentaneamente, as falhas nas barreiras se alinham, isso possibilita que um acidente ocorra.

Falhas ativas e falhas latentes correspondem aos fatores contribuintes de um incidente. Segundo a Cisp, fatores contribuintes são as circunstâncias, ações ou influências que desempenham um papel na origem ou no desenvolvimento de um incidente ou no aumento do risco de incidente. (RUNCIMAN et al., 2009) Alinhados aos conceitos de Reason (2000), Lawton e demais autores (2012) desenvolveram um referencial explicativo de fatores contribuintes de incidentes no ambiente hospitalar, intitulado o Modelo de Yorkshire. Esse modelo destaca cinco conjuntos de fatores: falhas ativas, falhas situacionais, condições do ambiente de trabalho, fatores organizacionais e fatores externos (Quadro 1). Além desses, os sistemas de comunicação e a cultura de segurança são fatores contribuintes que influenciam todos os demais. (LAWTON et al., 2012) 
Quadro 1 - Fatores contribuintes de incidentes no Modelo de Yorkshire

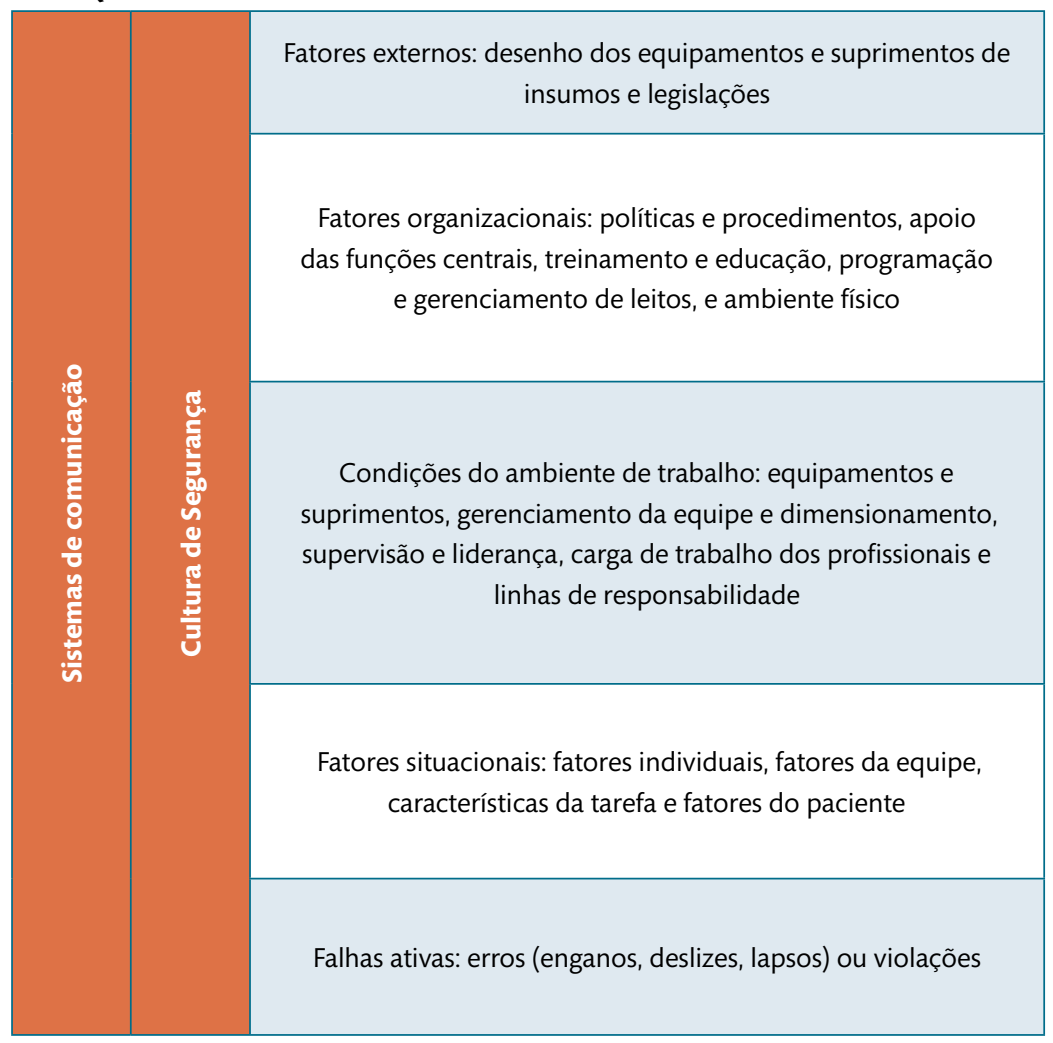

Fonte: elaborado pelas autoras com base em Lawton e colaboradores (2012).

Destacamos aqui o termo "cultura de segurança” pela sua importância e, também, pela forte presença nos discursos atuais para melhoria da segurança do paciente. Cultura de segurança - uma parte da cultura organizacional (VOGUS; SUTCLIFFE; WEICK, 2010) - consiste no conjunto de valores, atitudes e padrões de comportamento compartilhado por um grupo com relação à segurança. (GREAT BRITAIN, 1993) Toda organização de saúde compartilha uma determinada cultura de segurança que pode ser mais forte-quando 
a segurança é priorizada em relação à produtividade, por exemplo - ou mais fraca - nesse caso, observa-se o oposto. A cultura de segurança pode variar dentro de uma organização, tanto entre diferentes departamentos, como entre os grupos profissionais. (SINGER; VOGUS, 2013; TAYLOR et al., 2011)

\section{Práticas seguras e o desafio para a sua implementação}

O reconhecimento da magnitude dos problemas de segurança do paciente levou à proposição de práticas para a redução da ocorrência de eventos adversos. Em 2005, um ano após o estabelecimento da Aliança Mundial para Segurança do Paciente, a OMS fez um chamado aos estados-membros com o lançamento do primeiro desafio global para segurança do paciente - "Assistência limpa é uma assistência segura", que teve como foco a higienização de mãos para prevenção e redução das Infecções Relacionadas à Assistência à Saúde (IRAS). O segundo desafio global, "Cirurgias seguras salvam vidas", foi lançado em 2007. (OMS, 2009) O terceiro desafio global, em 2017, intitulado "Medicação sem risco", direciona-se para a redução dos danos associados aos medicamentos, causados por prática insegura e erros. (WHO, 2017) O Brasil é representado nos acordos com a OMS pela Agência Nacional de Vigilância Sanitária (Anvisa), que coordenou a tradução dos desafios globais para o português.

Além do estímulo ao desenvolvimento de iniciativas nacionais, a OMS propôs, em 2007, práticas locais para tornar o cuidado mais seguro, as chamadas Soluções para Segurança do Paciente, desenvolvidas pela Joint Commission e Joint Commission International

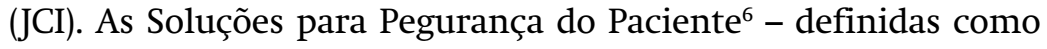

6 Ver em: https://www.who.int/patientsafety/topics/solutions/en/. 
qualquer redesenho do sistema ou intervenção que demonstre a capacidade de evitar ou mitigar dano ao paciente oriunda de processos de cuidado de saúde -, propostas pela OMS, incluem, além dos tópicos abordados nos desafios globais, a promoção da comunicação adequada durante a transferência do cuidado; evitar conexões erradas de cateter e tubo endotraqueal; e o uso de dispositivos para injeção uma única vez. (OMS, 2007)

Outra iniciativa da JCI que é bastante difundida e conhecida no Brasil é o conjunto de Metas Internacionais de Segurança do Paciente (Misp). As Misp ${ }^{7}$ fazem parte dos padrões para acreditação hospitalar pela JCI desde 2008, são elas:

1. Identificar paciente corretamente;

2. Melhorar a efetividade da comunicação;

3. Melhorar a segurança das medicações de alta vigilância;

4. Garantir cirurgia segura;

5. Reduzir o risco de IRAS; e

6. Reduzir o risco de dano ao paciente decorrente de queda.

A Agency for Healthcare Research and Quality (AHRQ) tem estimulado a análise crítica de práticas para segurança do paciente. Em 2013, publicou relatório influente com revisão da evidência científica sobre a efetividade de um conjunto de práticas, resultando em uma lista de 10 práticas fortemente recomendadas (Quadro 2).

7 Ver em: https://www.jointcommissioninternational.org/standards/ international-patient-safety-goals/. 
Quadro 2 - Práticas de segurança do paciente fortemente recomendadas segundo AHRQ, 2013

1. Lista de abreviações perigosas que não devem ser usadas;

2. Barreiras de precaução para a prevenção de infecções relacionadas à assistência à saúde;

3. Intervenções para melhorar a adesão à higienização das mãos;

4. Redução do uso desnecessário de cateter vesical e outras estratégias para prevenir infecções do trato urinário relacionada a cateter;

5. Prevenção de infecções da corrente sanguínea associadas a cateter venoso central;

6. Prevenção de pneumonia associada à ventilação mecânica;

7. Listas de verificação pré-operatória e de anestesia para prevenir incidentes relacionados à segurança na cirurgia, como infecções de sítio cirúrgico e cirurgia em local errado;

8. Uso de ultrassonografia em tempo real para guiar a inserção de cateter venoso central para aumentar a proporção de cateteres posicionados corretamente na primeira tentativa;

9. Intervenções multicomponentes para prevenir úlceras por pressão;

10. Estratégias para aumentar a profilaxia adequada do tromboembolismo venoso.

Fonte: elaborado pelas autoras com base em Mendes Júnior e Noronha $\left(2013\right.$, p. 56). ${ }^{8}$

Nos últimos 10 anos, pesquisadores têm reconhecido a necessidade de dedicar atenção ao contexto e ao processo de implementação das práticas seguras. A implementação incompleta ou desvirtuada de uma prática segura reduz a sua efetividade. Há estudos que mostram que práticas seguras com evidência de impacto positivo não apresentaram os mesmos efeitos quando implementadas em outros cenários. Os resultados impressionantes do projeto colaborativo de Michigan (PRONOVOST et al., 2006) não foram replicados no programa inglês para redução das infecções primárias de corrente sanguínea em Unidades de Terapia Intensiva (UTI).

8 Tradução dos autores baseada na Tabela C do Sumário Executivo do relatório Making Health Care Safer II. 
(DIXON-WOODS et al., 2013) De modo semelhante, redução de complicações pós-operatórias obtida no estudo multicêntrico de Haynes e demais autores (2009) não foi identificada após introdução da lista de verificação de segurança cirúrgica nos hospitais de Ontário, Canadá. (URBACH et al., 2014)

Não se pode assumir que a introdução de uma prática segura automaticamente levará a melhoria dos processos clínicos. (AVELING; MCCULLOCH; DIXON-WOODS, 2013) Atenção aos desafios socioculturais é fundamental para implementação efetiva de práticas seguras. (BOSK et al., 2009) A cultura de segurança é reconhecida como fundante para os esforços para melhoria da segurança do paciente. A centralidade da cultura está relacionada à natureza organizacional e sistêmica dos erros no cuidado de saúde (RAMANUJAM; ROUSSEAU, 2006; SHORTELL; SINGER, 2008): erros surgem de estruturas e processos organizacionais complexos e interrelacionados, que são realizados por meio de ações e interações diárias e contínuas de seus membros. (FELDMAN; ORLIKOWSKI, 2011)

\section{Aspectos políticos-institucionais da segurança do paciente no Brasil}

A segurança do paciente é um "princípio fundamental do cuidado em saúde” (WHO, 2017), que requer do Estado políticas para minimização dos riscos e erros relacionados com o cuidado assistencial, bem como atualização dos instrumentos de regulação e controle sanitário do uso das tecnologias utilizadas e associadas com danos aos pacientes.

Nesse sentido, a regulação é importante para monitorar e melhorar a segurança dos cuidados de saúde, com o objetivo de garantir tratamento seguro e confiável para pacientes e ambiente seguro para profissionais. (OIKONOMOW et al., 2019) No Brasil, 
a Vigilância Sanitária de Serviços de Saúde ocupa importante papel há décadas, não só formulando regulamentos e normas técnicas que visam à qualidade e à segurança na atenção à saúde, mas também monitorando a adesão das organizações a esses regramentos.

Além das iniciativas de cunho regulatório, existem também, no país, ações que podem ser reconhecidas como precursoras das ações de segurança em saúde, entre estas, estão os processos de certificação/acreditação, a implantação dos Núcleos de Qualidade em Serviços de Saúde, o Projeto Rede/Hospitais Sentinela, criado em 2002, pela Anvisa, a Rede Brasileira de Enfermagem e Segurança do Paciente (Rebraensp), fundada em 2008, o Portal Proqualis, iniciado em 2009 pela Fundação Oswaldo Cruz (Fiocruz), o Projeto de Avaliação de Desempenho de Sistemas de Saúde, o Programa Nacional de Melhoria do Acesso e da Qualidade da Atenção Básica, dentre outros.

O Brasil se engajou nos desafios globais em prol da segurança do paciente, na década de 2000. A Anvisa lançou duas importantes campanhas mundiais no país: "Uma assistência limpa é uma assistência mais segura" e "Cirurgias seguras salvam vidas", em 2007 e 2010, respectivamente. (BRASIL, 2013c) Em 2011, foi publicada a Resolução da Diretoria Colegiada (RDC) $n^{\circ} 63$, que pode ser considerada o primeiro marco regulatório nacional em prol da segurança do paciente, trata das "Boas práticas de funcionamento em serviços de saúde”, com um capítulo específico sobre segurança do paciente. (ANVISA, 2011) No entanto, as ações regulatórias tomaram maior visibilidade quando o Ministério da Saúde (MS) instituiu o Programa Nacional de Segurança do Paciente (PNSP) com a publicação da Portaria do Gabinete Ministerial (GM) n ${ }^{\circ} 529$ de $1^{\circ}$ de abril de 2013.

Esse programa visa organizar as iniciativas voltadas para a melhoria da qualidade da assistência e do cuidado à saúde prestados em todos os estabelecimentos de saúde do país. Suas atividades 
estão voltadas para estimular: a adesão às práticas assistenciais seguras, a pesquisa e o ensino sobre a temática; e o envolvimento do cidadão na sua segurança. (BRASIL, 2013c) Como estratégias estão definidas a obrigatoriedade de: criação de Núcleos de Segurança do Paciente (NSP); elaboração de planos locais de segurança do paciente nos estabelecimentos de saúde; implementação de protocolos; e notificação de incidentes relacionados à segurança do paciente. Outra importante iniciativa foi a criação, no âmbito do MS, do Comitê de Implementação do Programa Nacional de Segurança do Paciente (CIPNSP). Essa instância colegiada, de caráter consultivo, contava com a participação de representantes do MS, Fiocruz, Agência Nacional de Saúde Suplementar (ANS), Conselho Nacional de Secretários de Saúde (Conass), Conselho Nacional de Secretarias Municipais de Saúde (Conasems), Conselhos profissionais, Organização Pan-Americana da Saúde (Opas), Instituições de Ensino Superior (IES) e Anvisa. Seu propósito era apoiar a implementação do programa (BRASIL, 2013c), no entanto, esse Comitê foi extinto em 2019. (BRASIL, 2019)

Em 2013, o MS editou as Portarias $n^{\circ} 1.377$ e $n^{\circ} 2.095$ que aprovam os protocolos básicos de segurança do paciente a serem implantados nos hospitais brasileiros:

1. Cirurgia segura;

2. Prática de higienização das mãos em serviços de saúde;

3. Prevenção de úlceras por pressão;

4. Prevenção de quedas em pacientes internados;

5. Identificação do paciente;

6. Segurança na prescrição, uso e administração de medicamentos. (BRASIL, 2013a, 2013b)

Para a instituição das ações de segurança do paciente em todos os serviços de saúde do país, a Anvisa expediu também, em 2013, a RDC n 36 que normatiza a criação dos NSP, órgãos internos dos serviços de saúde, responsáveis pela condução das ações de 
segurança do paciente, por meio de um plano de ações, elaborado segundo especificidades de cada serviço, o qual deve estabelecer estratégias para a gestão de risco incluindo, a identificação, análise e monitoramento dos riscos e incidentes. (ANVISA, 2013)

Essa normativa se aplica aos serviços de saúde, de qualquer natureza, incluindo aqueles que exercem ações de ensino e pesquisa, sendo excluídos os consultórios individualizados, laboratórios clínicos, serviços móveis e de atenção domiciliar. Para a constituição dos NSP, a direção do serviço de saúde pode utilizar uma estrutura já existente no formato de comitês, comissões, gerências, ou coordenações e dentre seus participantes, destacar um profissional responsável com participação nas instâncias deliberativas do serviço de saúde. Dentre as competências dos NSP, a RDC no 36 determina a obrigatoriedade mensal da notificação de EA ocorridos nos serviços de saúde, ao Sistema Nacional de Vigilância Sanitária (SNVS), por meio do módulo Assistência à Saúde do Sistema de Notificações em Vigilância Sanitária (Notivisa). (ANVISA, 2013)

Em 2015, a Anvisa publicou o Plano Integrado para a Gestão Sanitária da Segurança do Paciente em Serviços de Saúde, com o objetivo de integrar as ações do SNVS, para a gestão da segurança do paciente em serviços de saúde do país, com competências descritas para os níveis federal, estadual e municipal da vigilância sanitária. (ANVISA, 2015)

Esse plano estabelece como prioridade o monitoramento de eventos adversos, compreende a notificação e a investigação como etapas essenciais da avaliação em serviços de saúde e sua operacionalização inicia-se pelos EA graves, IRAS e eventos que resultaram em óbito em todas as unidades dos serviços de saúde. Além disso, reforça e estimula a avaliação das práticas de segurança em serviços de saúde que possuem leitos de UTI, numa tentativa de trabalho em rede em prol da segurança em saúde no Brasil. (ANVISA, 2015) 
A partir da análise das notificações de eventos adversos do Notivisa, a Anvisa tem elaborado e divulgado relatórios com dados de eventos adversos das instituições de saúde brasileiras. O último relatório, com dados de junho de 2019 a maio de 2020, revela que os serviços de saúde das regiões Sudeste, Nordeste e Sul são os que mais notificam seus EA, com percentuais de 38,9, 20,7 e $20 \%$, respectivamente. Dentre os EA notificados, falhas durante a assistência à saúde, úlcera por pressão, fatores envolvendo cateter venoso, queda do paciente e falhas na identificação do paciente, são os mais frequentes. (ANVISA, 2019-2020)

A despeito das iniciativas e normativas sobre segurança do paciente vigentes no país desde 2011, alguns estudos nacionais apontam que esforços maiores para a melhoria de processos de trabalho em prol da segurança do paciente se fazem necessários no país.

Estudo que avaliou a implementação do PNSP em hospitais de grande porte da cidade de Salvador (BA) identificou que a maioria dos núcleos de segurança do paciente estudados nesses hospitais são formalmente constituídos, possuem planos de segurança do paciente, entretanto, apenas metade desses núcleos contam com profissional com dedicação exclusiva. Dos hospitais de grande porte estudados, metade deles implementaram todos os protocolos obrigatórios. Os autores concluíram que os NSP das instituições estudadas não atendem totalmente às políticas regulatórias vigentes no país, carecendo, portanto, de adequações e de controle sanitário efetivo. (COSTA et al., 2020)

Outro estudo sobre a implementação das atividades do PNSP em hospitais públicos brasileiros, apresentou a diversa realidade dessas unidades, inclusive as condições precárias em muitas delas. Entretanto, indica potencialidades para o fortalecimento da cultura de segurança, como acreditação e outros motivadores externos e, internamente, liderança inspiracional e equipes com quantitativo 
e treinamento adequados, uma vez que esses aspectos exercem influência sobre a execução de práticas seguras. (CALDAS, 2017)

\section{Breve panorama acerca da literatura científica sobre segurança do paciente na vigência da pandemia da COVID-19}

A pandemia da COVID-19, entre muitas consequências, impactou na produção científica no mundo. Após nove meses do seu início, o número de publicações ultrapassou a 100 mil, nos mais variados formatos e, na temática da segurança do paciente, são mais de 2 mil publicações. ${ }^{9}$ A distribuição diversa de objetos relacionados à segurança do paciente no curso da pandemia é acompanhada de dúvidas, incertezas, lacunas e avanços de conhecimentos científicos.

Nos primeiros cinco meses da pandemia, as produções foram voltadas para procedimentos específicos e, em especial, aqueles que envolvem a geração de aerossóis, com a manipulação da região da boca e nariz como traqueostomia, procedimentos otorrinolaringológicos e ventilação não invasiva. Procedimentos cirúrgicos de emergência também tiveram destaque com ênfase no treinamento da equipe e no uso adequado de Equipamentos de Proteção Individual (EPIs). Uso de máscara N95, óculos ou protetor de face, luvas duplas para a manipulação de vias áreas; planejamento no transporte de pacientes; e sala específica para cirurgia, de paciente sintomáticos e assintomáticos. O planejamento da cirurgia relacionada ao espaço físico, o sistema de ventilação adequado, a anestesia, as recuperações anestésicas foram também enfatizados. (SOUTO et al., 2020)

9 Ver: https://app.dimensions.ai/discover/publication?search_mode=content\& search_text=covid-19\&search_type=kws\&search_field=text_search. 
Além da produção voltada para diagnóstico e tratamento, um conjunto de estudos e recomendações acerca de riscos relacionados aos ambientes dos serviços de saúde, abordagens organizacionais para gerenciamento do risco e aumento da segurança para pacientes e profissionais, apontando uma tendência de abordar a segurança do paciente de forma ampliada, como segurança em saúde. Assim, a organização do espaço físico nos serviços de saúde, distância física, etiqueta da tosse, uso de máscara, limite de pessoas em elevadores e áreas comuns, monitoramento de entradas/ saídas, espaços exclusivos para pacientes com COVID-19, ventilação adequada com filtragem por filtros HEPA - filtro de ar particulado de alta eficiência - ou boa ventilação e visitas limitadas foram também objeto de estudos e reflexões. (SOUTO et al., 2020)

A partir de junho, esses temas permanecem e cresce o número de publicações relacionada às terapias farmacêuticas, abordando principalmente a cloroquina e hidroxicloroquina e o plasma convalescente. A telemedicina e telessaúde para consultas e procedimentos diversos ganham destaque, recomendado o seu uso para questões relacionadas desde à saúde mental até outras especialidades médicas. Essa transição de consultas presenciais para o contato remoto é ressaltada nos estudos, como medida de segurança configurando uma mudança radical nas formas e na relação dos profissionais com os usuários dos serviços de saúde. Não foram identificados trabalhos relacionados aos modelos de atenção em saúde, mas a segurança do paciente surge na atenção primária, embora com poucas publicações. Continuam presentes na literatura científica os serviços cirúrgicos, assim como procedimentos em geral, localizados na cabeça, e cresce a produção relacionada à oftalmologia.

O pouco conhecimento sobre o SARS-CoV-2, agente causador da doença, assim como da sua atuação no organismo humano tem sido, aos poucos, superado. A identificação de questões fundamentais 
sobre o vírus e a doença, como a carga viral necessária para produzir a infecção e o caráter inflamatório e sistêmico da doença, é de grande relevância no controle de riscos nos serviços de saúde e no cuidado em saúde. A forma como o diagnóstico e a terapêutica da COVID-19 foram sendo modificados, o que reflete o desconhecimento, as incertezas e inseguranças. A ausência de medicamento específico para o tratamento suscitou uma corrida em busca de respostas que se traduziram em expressiva realização de ensaios clínicos, sejam aqueles testando novos medicamentos como outros buscando novos usos para aqueles já conhecidos. Após seis meses do início da pandemia, mais de 500 ensaios clínicos estavam sendo conduzidos em todo mundo e 265 medicamentos estavam sob investigação. (IDDA; SORU; FLORIS, 2020) Embora esses estudos sejam de grande relevância e possam trazer luz para o tratamento da doença, podem também contribuir com a ocorrência de eventos adversos e afetar negativamente a segurança do paciente.

Além da publicação em periódicos, esse tema tem sido bastante abordado por autoridades sanitárias em todo mundo. São orientações, alertas e vários materiais de apoio para os profissionais e serviços de saúde. No Brasil, a Anvisa tem elaborado notas técnicas para subsidiar a organização e as práticas visando à melhoria da qualidade do cuidado e da segurança do paciente.

As secretarias de saúde de estados e municípios, assim como instituições de pesquisas - Fiocruz, Instituto Nacional do Câncer (Inca), Rede de Laboratórios em Saúde Pública, Instituto Nacional de Controle de Qualidade em Saúde (INCQS) - e associações profissionais também têm contribuído com a produção de conhecimento voltado para a segurança do paciente em tempos de COVID-19. 


\section{Contribuições para definição de estratégias organizacionais para a gestão do risco durante a pandemia da COVID-19}

A partir da visão do que foi produzido e publicado sobre segurança do paciente e a COVID-19 nesses meses, foram identificados alguns aspectos considerados relevantes no que tange à adoção de estratégias por parte das organizações de saúde para o enfrentamento da pandemia. Além das medidas de proteção, inciativas de reorganização e adaptação da gestão, de ambientes e de processos de trabalho, a literatura chama a atenção para a necessidade de que, frente a um contexto de incertezas e de mudanças rápidas, é preciso pensar para além das abordagens de gestão de risco, e também refletir sobre como tornar as organizações de saúde mais resilientes. (RANGACHARI; WOODS, 2020)

Organizações de saúde são reconhecidas como extremamente complexas. (MINTZBERG, 2003) Há uma diversidade de processos de trabalho, centrados no profissional de saúde que precisa, por um lado, conhecer e seguir protocolos e, por outro, ter um grau de autonomia razoável e criatividade para adaptar o trabalho às necessidades dos pacientes e de novas circunstâncias. Essas características, associadas aos diversos perigos e riscos relacionados à assistência à saúde já bastante conhecidos, tornam as organizações de saúde lugares inseguros, que precisam se preparar para gerenciá-los da forma mais adequada.

Na vigência da pandemia da COVID-19, ainda que um percentual pequeno de indivíduos acometidos precise de internação, em números absolutos, houve, em todo o mundo, um aumento abrupto da demanda por atendimento que sobrecarregou todos os sistemas de saúde. A assistência a esses pacientes, como pode ser visto, requer cuidados intensivos, envolvendo procedimentos que expõem os profissionais a maior risco de contaminação. Desse 
modo, a situação envolve profissionais sobrecarregados, lidando com um vírus altamente transmissível, em estruturas improvisadas, em um contexto de escassez de materiais e de insumos necessários para uma prática efetiva e segura. Essas circunstâncias levam a desgaste, medo e angústia e, nesse cenário, os gestores e lideranças das organizações de saúde precisam adotar rapidamente medidas para proteger suas equipes e dar ordem ao caos que se instala de repente.

Este tem sido o desafio posto pela pandemia da COVID-19: fornecer cuidados efetivos e seguros a um volume de pacientes muito acima daquele a que as organizações costumam atender, resguardando a segurança também dos profissionais de saúde, não apenas em relação ao vírus, mas considerando o impacto na sua condição emocional. Assim, as práticas seguras e as soluções para segurança do paciente, já mencionadas, foram objeto de notas técnicas e recomendações para que fossem reforçadas.

As organizações de saúde precisaram ser capazes de lidar com o impacto da situação e se adaptarem. No entanto, sabe-se que a implementação de mudanças pontuais não parece ser suficiente, toda a concepção da provisão do cuidado precisa ser reorganizada e pensada de modo sistêmico. Afinal, Reason (2000) afirma que a segurança do paciente depende de uma abordagem sistêmica. Isso inclui ter uma visão estratégica para a organização que atenda às necessidades de seus trabalhadores e de seus pacientes, apoiada em uma comunicação clara e eficaz.

Na vigência da pandemia da COVID-19, esse desafio pareceu ser menos ameaçador para aquelas organizações de saúde que já contavam com uma boa base de gestão, informação rápida e confiável, mecanismos de comunicação, estrutura física adequada, sistema de logística ajustado, fluxos e processos de trabalho bem desenhados. Esses são elementos que permitem conjugar a capacidade de antecipação com a de improvisação, para definir um 
conjunto de intervenções de cunho organizacional. Em outras palavras, tornar a organização resiliente.

A resiliência organizacional consiste essencialmente na capacidade de entender a magnitude das crises e de seus impactos potenciais e prever e mitigar riscos antes que eles prejudiquem a organização. A resiliência deve ser um traço da cultura de uma organização para que ela possa sobreviver e superar uma crise. (DOMINGUEZ JR, 2020) Por isso, o papel das lideranças é apontado como fundamental. É por meio delas que se dá a coordenação do trabalho, que precisa envolver a equipe e adotar uma comunicação clara e aberta entre todos os envolvidos na organização e prestação da assistência, para propiciar esse entendimento amplo que otimiza esforços e recursos. O resultado desse processo é aprendizado coletivo que torna a organização capaz de se adaptar, isto é, ser resiliente. (RERUP, 2001)

Quanto mais os gestores, as lideranças e os profissionais conhecerem sua organização e dominarem competências, entre elas, a capacidade de escutar, mais elementos terão para promover as mudanças e adaptações para fazer frente às novas demandas. Profissionais de todos os setores da organização precisam estar envolvidos nesse processo para que a criatividade e a improvisação estejam pautadas no conhecimento real e profundo da organização. Para ter uma visão mais integrada da organização, ancorada na abordagem sistêmica adotada na segurança do paciente, conforme o proposto por Reason (2000), o gestor e sua equipe podem tomar o Modelo de Yorkshire, anteriormente apresentado, para orientar sua ação. Desse modo, improvisar torna-se um exercício calculado, antecipando chances de erros e acertos.

A constituição de um comitê com representantes dos diferentes setores pode auxiliar a ter a visão global dos impactos e possibilidades de superação. Além de mapear riscos e fragilidades, pode apontar as potencialidades e permitir pensar intervenções 
de forma mais integrada, compreendendo a imbricada relação entre as partes dessa organização, antecipando os impactos das mudanças em todos os seus setores. Um comitê ativo, que participe de todo o processo de planejamento da organização, inclusive do monitoramento do plano de intervenção, pode identificar oportunamente resultados que, se distintos daqueles esperados, levem a revisão rápida da intervenção, com adoção de medidas de correção em tempo hábil. Esse comitê pode ainda ajudar na estratégia de comunicação, mantendo um canal aberto informando sobre as decisões e ouvindo suas repercussões nos diferentes setores da organização. (GUPTA; FEDERMAN, 2020)

As linhas mestras para as mudanças são aquelas que tradicionalmente orientam o processo de enfrentamento de crises e emergências. Mapear os riscos e antecipar como serão afetados, pois a ideia é reduzi-los de forma sustentável; redesenhar processos e adaptar espaços físicos, estabelecendo barreiras para contenção dos riscos; capacitar equipes por meio de atividades coletivas, inclusive utilizando simulações realísticas e reforçando protocolos, adaptando-os para às novas necessidades; prover o material e outros recursos necessários; proteger os mais vulneráveis, o que implica em afastamento ou trabalho remoto para alguns indivíduos; estabelecer escalas adaptadas ao desgaste físico e emocional; estabelecer uma linha de comunicação ágil e clara, envolvendo toda a organização; monitorar, refletir e corrigir. É preciso salvar vidas, observando a segurança de todos e a estabilidade da organização. (LLOYD-SMITH, 2020)

Assim, o gerenciamento de situações complexas dentro da área de saúde requer atenção às ameaças emergentes em uma perspectiva mais ampla da organização, isso inclui tanto pacientes como profissionais de saúde. Não foi por acaso que ganhou ampla repercussão o tema da saúde do trabalhador da saúde e sua importância para a segurança do paciente. (ADAMS; WALLS, 2020; TEIXEIRA et al., 2020) 
O processo de adaptação da organização pode implicar também na mudança da estrutura de liderança, no entanto, as referências já estabelecidas e reconhecidas não devem ser desprezadas. Esses profissionais são importantes elos e disseminam a cultura de segurança do paciente na organização. Atuam no monitoramento e na promoção da adesão aos protocolos e às práticas seguras; são as primeiras pessoas a lidar com a ocorrência de incidentes, acolhem os profissionais e observam aspectos da segurança dos trabalhadores da saúde, propondo inclusive práticas educativas e de apoio para prevenir exposições e para lidar com o stress.

Esse esforço de reorganização deve ser amplamente divulgado para toda a organização, estendendo-se também para os usuários. Há várias medidas de proteção que precisam ser bem entendidas, seus fundamentos e repercussões sobre cada indivíduo e sobre a coletividade.

Não há um único modelo de ações relacionadas à segurança do paciente a ser adotado pelas organizações de saúde para o enfrentamento da COVID-19. Cada organização tem que definir suas necessidades e capacidades, no entanto, prover infraestrutura e materiais adequados e suficientes, promover a adesão às práticas seguras, motivar e cuidar dos profissionais e engajar os pacientes parece ser um caminho promissor.

\section{Reflexões acerca dos efeitos da pandemia na segurança do paciente}

A pandemia da COVID-19 tornou evidente que o Sistema Único de Saúde (SUS) é uma das maiores conquistas e patrimônio dos brasileiros. Apesar da falta de um plano nacional, que conjugasse os esforços das três esferas de gestão para seu enfrentamento, o acesso de um contingente expressivo da população acometida 
a um conjunto diverso de intervenções - prevenção, proteção, promoção e assistência -, está sendo garantido.

No entanto, é forçoso reconhecer que, como a segurança do paciente depende do quão bem organizados e funcionantes estão os vários componentes do cuidado de saúde, referir-se a segurança do paciente parece algo distópico. O desfinanciamento crônico do SUS tem implicado em problemas de qualidade, com estrutura e condições de trabalho insatisfatórias nos serviços de saúde, falta de profissionais ou com vínculos de trabalho precários, que não permitem nem mesmo a formação de equipes.

Existe hoje conhecimento acerca das práticas de segurança em saúde que podem reduzir os erros, prevenir incidentes e EA. São medidas que salvam vidas e melhoram a qualidade do cuidado em saúde. Entretanto, sua implementação e a adesão dos profissionais de saúde a elas ainda são um grande desafio em todo o mundo. Para isso, são necessárias mudanças na cultura organizacional, nos processos e atividades em todos os níveis do cuidado assistencial, ancoradas em uma consistente política nacional e de financiamento do SUS, e seus desdobramentos até o nível de cada unidade de saúde. No caso da segurança do paciente, tal política precisa estar voltada não apenas para a regulação dos processos, mas para o monitoramento e avaliação das atividades desenvolvidas nas esferas respectivas de gestão. (WHO, 2014)

A despeito do arcabouço regulatório constituído no Brasil, certamente um avanço normativo, no contexto da pandemia, cabe refletir se tal arcabouço tem sido suficiente para o enfrentamento da situação, se as estratégias contribuíram para fortalecer os NSP e se a segurança dos profissionais de saúde está sendo garantida.

Para além do conhecimento e cumprimento dos processos regulatórios, requer-se a implementação do PNSP para a qual são necessárias condições estruturais, estratégias político-administrativas e planejamento das ações. Esse programa pode ser 
aprimorado adotando a visão ampliada de segurança do paciente trazida pela OMS em documento recente. Nessa revisão conceitual, a segurança do paciente é apresentada como um conjunto amplo de atividades que visam criar culturas e estimular comportamentos, estabelecer processos e procedimentos, adotar tecnologias e promover ambientes em que se busque de forma consistente e sustentável reduzir riscos, EA, e seus impactos. (WHO, 2020a)

A pandemia parece ter dado maior visibilidade aos temas da segurança do paciente e qualidade do cuidado. O desafio posto é que cada país deve ser capaz de se organizar para oferecer cuidados de qualidade e seguros para todos. Práticas seguras, cuidados efetivos e redesenho de políticas, programas e organizações a fim de garantir acesso com qualidade.

Assim, cabe refletir a importância de adotar estratégias no Brasil para harmonizar condutas pelas instâncias de governo, respeitadas as devidas autonomias, especificidades e perfil epidemiológico em cada território, uniformizar procedimentos assistenciais pautados na ciência, alocar de forma equânime recursos humanos e de infraestrutura, de modo transparente, integrado, descentralizado em conformidade com os princípios norteadores do sistema nacional de saúde brasileiro.

\section{Referências}

ADAMS, J. G.; WALLS, R. M. Supporting the Health Care Workforce During the COVID-19 Global Epidemic. JAMA, Chicago, v. 323, n. 15, p. 1439-1440, 2020.

AGÊNCIA NACIONAL DE VIGILÂNCIA SANITÁRIA (Brasil) - ANVISA.

Plano Integrado para a Gestão Sanitária da Segurança do Paciente em Serviços de Saúde: monitoramento e investigação de eventos adversos e avaliação de práticas de segurança do paciente. Brasília, DF: ANVISA, 2015. 
AGÊNCIA NACIONAL DE VIGILÂNCIA SANITÁRIA (Brasil) - ANVISA. RDC n³6. Institui ações para a Segurança do Paciente em Serviços de Saúde. Diário Oficial da União, Brasília, DF, 26 jul. 2013.

AGÊNCIA NACIONAL DE VIGILÂNCIA SANITÁRIA (Brasil) - ANVISA. Resolução RDC $n^{\circ}$ 63. Dispõe sobre os Requisitos de Boas Práticas de Funcionamento para os Serviços de Saúde. Diário Oficial da União, Brasília, DF, 25 nov. 2011.

AGÊNCIA NACIONAL DE VIGILÂNCIA SANITÁRIA (Brasil) - ANVISA. Relatório de incidentes relacionados à assistência à saúde: resultados das notificações realizadas no NOTIVISA. Brasil. Brasília, DF, 2019-2020.

AVELING, E.-L.; MCCULLOCH, P.; DIXON-WOODS, M. A qualitative study comparing experiences of the surgical safety checklist in hospitals in high-income and low-income countries. BMJ open, London, v. 3, n. 8, p. e003039, 2013.

BOSK, C. L. et al. Reality check for checklists. Lancet, London, v. 374, n. 9688, p. 444-445, Aug. 2009.

BRASIL. Decreto ${ }^{\circ} 9.759$, de 11 de abril de 2019. Extingue e estabelece diretrizes, regras e limitações para colegiados da administração pública federal. Diário Oficial da União: seção 1, Brasília, DF, n. 70-A, p. 5, 2019.

BRASIL. Ministério da Saúde. Portaria n 1.377, de 9 de julho de 2013. Aprova os Protocolos de Segurança do Paciente. Diário Oficial da União, Brasília, DF, 9 jul. $2013 a$.

BRASIL. Ministério da Saúde. Portaria nº. 2.095. Aprova Protocolos de Segurança. Diário Oficial da União, Brasília, DF, 24 set. 2013b.

BRASIL. Ministério da Saúde. Portaria n 529, de $1^{\circ}$ de abril de 2013. Institui o Programa Nacional de Segurança do Paciente (PNSP). Diário Oficial da União, Brasília, DF, 2 abr. 2013c.

CALDAS, B. N. Implementação do Programa Nacional de Segurança do Paciente em hospitais públicos: uma avaliação qualitativa. 2017. Tese (Doutorado em Saúde Pública) - Escola Nacional de Saúde Pública Sergio Arouca, Fundação Oswaldo Cruz, Rio de Janeiro, 2017.

COSTA, E. A. M. et al. Segurança do paciente em Serviços de Saúde: uma análise na cidade de Salvador, BA. Revista SOBECC, São Paulo, v. 25, n. 1, p. 17-24, 2020.

COUTO R. C.; PEDROSA, T. M. G, ROSA, M. B. Erros acontecem: construindo um sistema de saúde mais seguro. Belo Horizonte: IESS, 2016. 
DIXON-WOODS, M. et al. Explaining Matching Michigan: an ethnographic study of a patient safety program. Implementation Science, [s. I.], v. 8, n. 1, p. 70, June 2013.

DOMINGUEZ.JR, O. J. From the Officer's Desk: Organizational Resilience Through COVID-19. EMSWorld, New Jersey, 16 ago. 2020.

FELDMAN, L. B. Gestão de risco na enfermagem. In: FELDMAN, L. B. (org.). Gestão de risco e segurança hospitalar. 2. ed. São Paulo: Martinari, 2009. p. 111-136.

FELDMAN, M. S.; ORLIKOWSKI, W. J. Theorizing Practice and Practicing Theory. Organization Science, Providence, RI, v. 22, n. 5, p. 1240-1253, 2011.

GREAT BRITAIN. Advisory Committee on the Safety of Nuclear Installations. ACSNI Study Group On Human Factors: Third report: organising for safety. Sheffield: HSE, 1993.

GUPTA, S.; FEDERMAN, D. G. Hospital preparedness for COVID-19 pandemic: experience from department of medicine at Veterans Affairs Connecticut Healthcare System. Postgraduate Medicine, New York, v. 32, n. 6, p. 489-494. 2020.

HAYNES, A. B. et al. A surgical safety checklist to reduce morbidity and mortality in a global population. The New England Journal of Medicine, Boston, v. 360, n. 5, p. 491-499, Jan. 2009.

IDDA, M. L.; SORU, D.; FLORIS, M. Overview of the First 6 Months of Clinical Trials for COVID-19 Pharmacotherapy: The Most Studied Drugs. Frontiers in Public Health, Lausanne, v. 21, n. 8, p. 497, Aug. 2020.

LAWTON, R. et al. Development of an evidence-based framework of factors contributing to patient safety incidents in hospital settings: a systematic review. BMJ Quality \& Safety, London, v. 21, n. 5, p. 369-380, May 2012.

LLOYD-SMITH M. The COVID-19 pandemic: resilient organisational response to a low-chance, high-impact event. BMJ Leader, London, n. 4, p. 109-112, 2020.

MAKARY, M. A; DANIEL, M. Medical error-the third leading cause of death in the US. BMJ, London, n. 353, p. i2139, 2016.

MENDES JÚNIOR, W. V.; NORONHA, J. (coord.). Qualidade de serviços de Saúde no SUS: relatório final. [S. I.: s. n.], dez. 2013. Disponível em: https://proqualis. net/sites/proqualis.net/files/Relat\%C3\%B3rio\%20Final\%20Qualisus\%2016\%20 DEZ_2013. pdf. Acesso em: 23 ago. 2020 
MENDES, W. Taxonomia em segurança do paciente. In: SOUSA, P.; MENDES, W. (ed.). Segurança do paciente: conhecendo os riscos nas organizações de saúde. 2 ed. rev. e ampl. Rio de Janeiro: CDEAD/ENSP/Fiocruz, 2019. p. 59-73.

MINTZBERG, H. Criando organizações eficazes. 2. ed. São Paulo: Atlas, 2003.

OIKONOMOW, E. et al. Patient safety regulation in the NHS: the regulatory landscape of healthcare. BMJ Open, London, v. 9, n. 7, p. e028663, 2019.

ORGANIZAÇÃO MUNDIAL DA SAÚDE - OMS. Cirurgias seguras salvam vidas (orientações para cirurgia segura da OMS): segundo desafio global para a segurança do paciente. Rio de Janeiro: Organização Pan-Americana da Saúde, 2009.

PRONOVOST, P. et al. An intervention to decrease catheter-related bloodstream infections in the ICU. The New England Journal of Medicine, Boston, v. 355, n. 26, p. 2725-2732, 2006.

RAMANUJAM, R.; ROUSSEAU, D. M. The challenges are organizational not just clinical. Journal of Organizational Behavior, Chichester, v. 27, n. 7, p. 811-827, Nov. 2006.

RANGACHARI, P.; WOODS, J. L. Preserving Organizational Resilience, Patient Safety, and Staff Retention during COVID-19 Requires a Holistic Consideration of the Psychological Safety of Healthcare Workers. International Journal of Environmental Research and Public Health, Basel, v. 17, n. 12, p. 4267, 2020.

REASON, J. Human error: models and management. BMJ, London, v. 320, n. 7237, p. 768-770, Mar. 2000.

REIS, C. Cultura de segurança em organizações de saúde. In: SOUSA, P.; MENDES, W. (ed.). Segurança do Paciente: criando organizações de saúde seguras. 2 rev. e ampl. ed. Rio de Janeiro: Escola Nacional de Saúde Pública, Educação à Distância : Editora Fiocruz, 2019. p. 77-109.

RERUP, C. 'Houston We Have a Problem': Anticipation and Improvisation as Sources of Organizational Resilience. Comportamento Organizacional e Gestão, Lisboa, v. 7, n. 1, p. 27-44, 2001.

RUNCIMAN, W. et al. Towards an International Classification for Patient Safety: key concepts and terms. Journal of the International Society for Quality in Health Care, Oxford, v. 21, n. 1, p. 18-26, Feb. 2009.

SCOTT, S.D. et al. The natural history of recovery for the healthcare provider "second victim" after adverse patient events. Qual Saf Health Care, London, v. 1, n. 5, p. 325-30, Oct. 2020. DOI: 10.1136/qshc.2009.032870. 
SHORTELL, S. M.; SINGER, S. J. Improving patient safety by taking systems seriously. JAMA, Chicago, v. 299, n. 4, p. 445-447, 2008.

SINGER, S. J.; VOGUS, T. J. Reducing hospital errors: interventions that build safety culture. Annual Review of Public Health, Palo Alto, v. 34, p. 373-396, 2013.

SOUTO, A. C. et al. Recomendações para procedimentos assistências em saúde à luz da segurança do paciente durante a pandemia de Covid-19. 2020. Disponível em: https://redecovida.org/main-site-covida/wp-content/uploads/2020/09/RelatorioSeguran\%C3\%A7a-do-Paciente.pdf. Acesso em: 23 nov. 2020.

TAYLOR, S. L. et al. What context features might be important determinants of the effectiveness of patient safety practice interventions? BMJ Quality \& Safety, London, v. 20, n. 7, p. 611-617, July 2011.

TEIXEIRA, C. F. de S. et al. The health of healthcare professionals coping with the Covid-19 pandemic. Ciência e Saúde Coletiva, Rio de Janeiro, v. 25, n. 9 , p. 3465-3474, 2020.

URBACH, D. R. et al. Introduction of surgical safety checklists in Ontario, Canada. The New England Journal of Medicine, Boston, v. 370, n. 11, p. 1029-1038, Mar. 2014.

VOGUS, T. J.; SUTCLIFFE, K. M.; WEICK, K. E. Doing No Harm: Enabling, Enacting, and Elaborating a Culture of Safety in Health Care. Academy of Management Perspectives Briarcliff Manor, NY, v. 24, n. 4, p. 60-77, Nov. 2010.

WORLD HEALTH ORGANIZATION - WHO. Patient Safety: Making health care safer. Geneva: WHO, 2017.

WORLD HEALTH ORGANIZATION - WHO. Global Patient Safety Action Plan 2021-2030 - Towards Zero Patient Harm in Health Care. [S. I.]: First Draft, 2020a.

WORLD HEALTH ORGANIZATION - WHO. Transmission of SARS-COV-2: implications for infection prevention precautions scientific brief. 9 July $2020 \mathrm{~b}$. Disponível em: https://www.who.int/news-room/commentaries/detail/ transmission-of-sars-cov-2-implications-for-infection-prevention-precautions. Acesso em: 23 nov. 2020.

WORLD HEALTH ORGANIZATION - WHO. Guide for Developing National Patient Safety Policy and Strategic Plan. Brazzaville: WHO, 2014.

WU, A. W. Medical error: the second victim: the doctor who makes the mistake needs help too. BMJ, London, v. 320, n. 7237, p. 726-727, 2000. 